\title{
Intricarene, An Unprecedented Trispiropentacyclic Diterpene from the Caribbean Sea Plume Pseudopterogorgia kallos
}

\author{
Jeffrey Marrero, Abimael D. Rodríguez, ${ }^{\star}$ Charles L. Barnes \\ Deparment of Chemistry, University of Puerto Rico, P.O. Box 23346, U.P.R. Station, \\ San Juan, Puerto Rico 00931-3346, Deparment of Chemistry, 123 Chemistry Building, \\ University of Missouri-Columbia, Columbia, Missouri 65211
}

\section{List of Supporting Information:}

(1) Detailed description of experimental procedures.

(2) Copies of ${ }^{1} \mathrm{H}$ - and ${ }^{13} \mathrm{C}-\mathrm{NMR}$ spectra for intricarene (1).

(3) Tables of crystal data for intricarene (1) (crystal data and structure refinement, atomic coordinates, bond lengths and angles, anisotropic displacement parameters and hydrogen coordinates). 


\section{Experimental Section}

General Experimental Procedures: 1D- and 2D-NMR spectral data were recorded with a Bruker DRX-500 FT-NMR spectrometer. Infrared spectra were obtained with a Nicolet Magna FT-IR 750. The optical rotation was obtained with an Autopol IV automatic polarimeter. Column chromatography was performed on silica gel (35-75 mesh) and TLC analyses were carried out using glass precoated silica gel plates. HPLC was performed using a Polar Bonded Cyano Ultrasphere semipreparative column $(5.0 \mathrm{~mm} \times 25 \mathrm{~cm})$. All solvents used were either spectral grade or were distilled from glass prior to use. The percentage yield of PkHVI.9crystal (1) is based on the weight of the hexane extract.

Collection of Pseudopterogorgia kallos: P. kallos (Bielschowsky) was collected by SCUBA at depths of 83-91 ft in Old Providence Island, Colombia, on March 15, 2002. A voucher specimen is stored in the Chemistry Department of the University of Puerto Rico, Río Piedras Campus.

Extraction and Isolation Procedure: The organism was partially sundried, frozen and lyophilized prior to its extraction. The dry organism (1.07 $\mathrm{kg}$ ) was blended using $\mathrm{CH}_{2} \mathrm{Cl}_{2}-\mathrm{MeOH}(1: 1)(20 \times 1 \mathrm{~L})$. After filtration the crude extract was evaporated and stored under vacuum to yield a green gum (166 g). The crude extract was partitioned between hexane and $\mathrm{H}_{2} \mathrm{O}$, $\mathrm{CHCl}_{3}$ and $\mathrm{H}_{2} \mathrm{O}$, and then the aqueous suspension was extracted with EtOAc $(2 \times 2 \mathrm{~L})$. The resulting hexane extract was concentrated in vacuo to yield $71.9 \mathrm{~g}$ of a green amorphous The hexane extract was dissolved in a small volume of toluene, filtered, and loaded into a large Bio-Beads SX-3 with toluene as a eluent. Seven fractions (I-VII) were obatained based on the TLC analysis. Fraction VI was separated into 19 fractions (1-19) by silica gel column chromatography using $20 \%$ of EtOAc in hexane as eluent. After refrigeration storage small crystals growth in the vial for fraction VI.9. The crystalline solid was washed with a cool solution of $15 \%$ of hexane in isopropanol and dryed in vacuum. After NMR analysis of the crystalline solid the pure compound $(1,4.0 \mathrm{mg}$, yield $=0.00556 \%$ ) was recrystallized in amixture of $\mathrm{MeOH} /$ actone $/ \mathrm{CHCl}_{3}$ gave crystals of excellent quallity for $\mathrm{X}$ ray analysis. 
intricarene (1): colorless crystalline solid; $[\alpha]^{20}{ }_{\mathrm{D}}+50.0^{\circ}\left(\mathrm{c} 0.7, \mathrm{CHCl}_{3}\right)$; UV $(\mathrm{MeOH}) \lambda_{\max } 245 \mathrm{~nm}$ ( $\varepsilon$ 6200); IR (thin film) 3078, 2947, 2925, 1767, $1690,1641,1627,1440,1380,1282,1184,1166,1002,973 \mathrm{~cm}^{-1} ;{ }^{1} \mathrm{H}$ NMR $\left(\mathrm{CDCl}_{3}, 500 \mathrm{MHz}\right)$ and ${ }^{13} \mathrm{C} \mathrm{NMR}\left(\mathrm{CDCl}_{3}, 125 \mathrm{MHz}\right.$ ) (see Table 1); EIMS $\mathrm{m} / \mathrm{z}$ $326\left(\mathrm{M}^{+}, 74\right), 311$ (23), 298 (13), 230 (100), 216 (40), 215 (46), 91 (33); HREIMS $m / z[M]^{+} 326.1524$ (calcd for $\mathrm{C}_{20} \mathrm{H}_{22} \mathrm{O}_{4}$ ).

X-ray Single-Crystal Structure Determination of intricarene (1). Crystal data: $\mathrm{C}_{20} \mathrm{H}_{22} \mathrm{O}_{4}, M_{r}=326.38$, monoclinic, space group $P 2_{1}, a=$ 7.1134(4), $b=12.6053(6), c=9.4510(5) \AA, \beta=105.5470(2)^{\circ}, V=816.43(6)$ $\AA^{3}, Z=2, \rho_{\text {calc }}=1.328 \mathrm{Mgm}^{-3}, F_{000}=348, \lambda\left(\mathrm{Mo}_{\mathrm{K \alpha}}\right)=0.71073 \AA, \mu=0.092$ $\mathrm{mm}^{-1}$. Data collection and reduction: crystal size, $0.50 \times 0.25 \times 0.25$ $\mathrm{mm}^{3}, \theta$ range, 2.24-27.16, 5909 reflections collected, 1882 independent reflections $\left(R_{\mathrm{int}}=0.0291\right)$, final $R$ indices $(I>2 \sigma(I)): R_{1}=0.0322, w R_{2}=0$ for 220 variable parameters, GOF $=1.030$. 


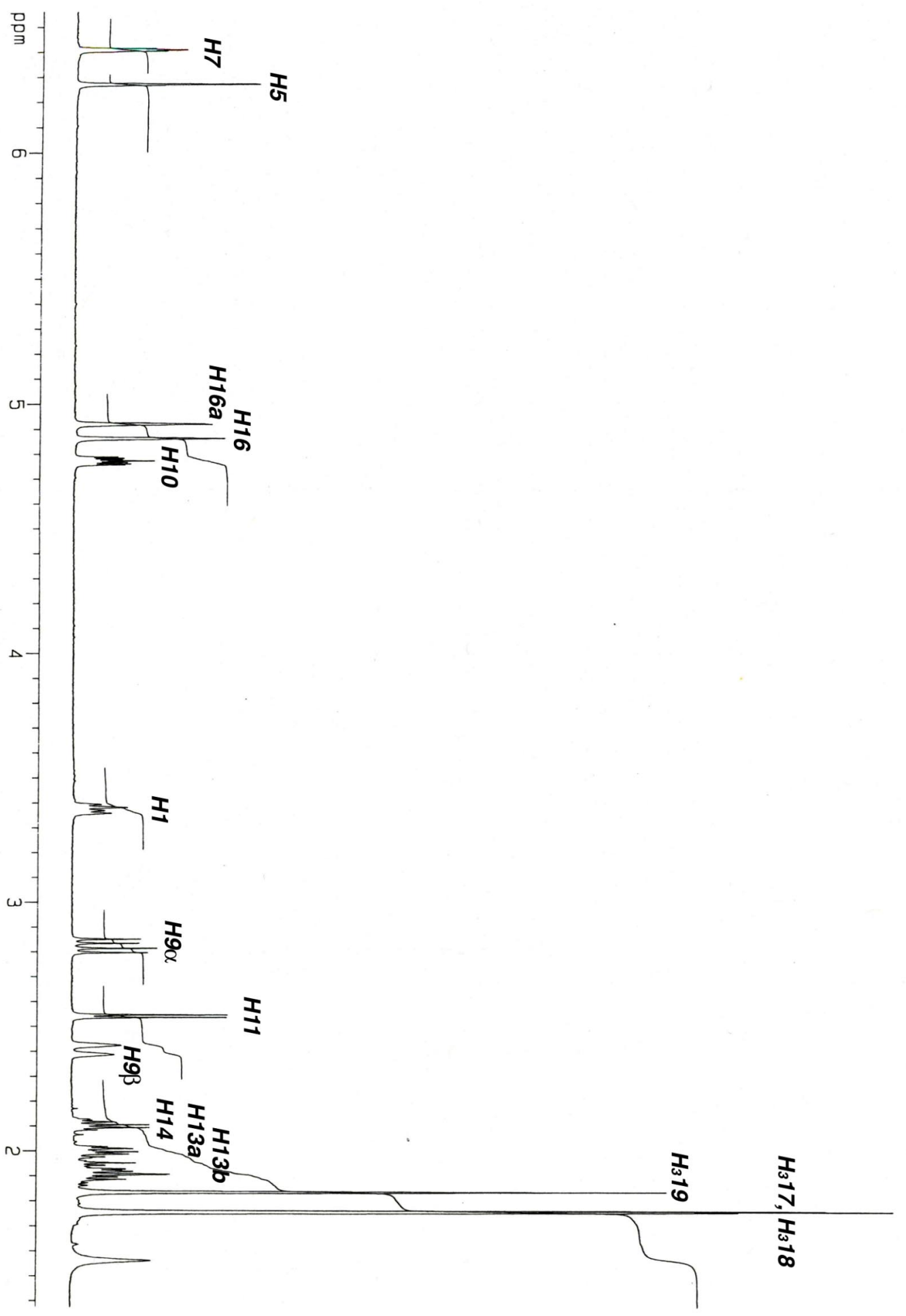

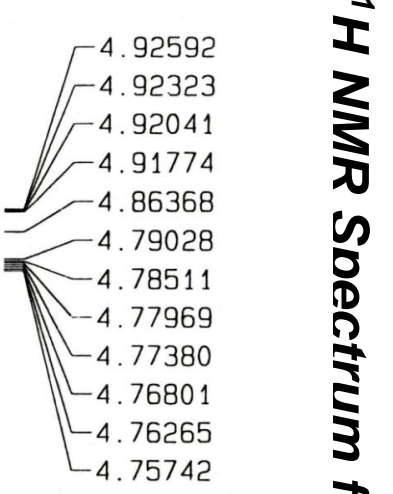

-3. 39499

-3. 38321

-3.37074

-3.35971

2.85241

- 2.83530

-2.81525

2. 79812

$-2.54814$

-2.53756

2.42539

-2.10678

$-2.09534$

] -2.01105

$=[-1.99832$

$-1.95388$

$-1.90865$

$-1.90648$

$=1.83872$

$\exists / 1.83632$

1.83399

1.76106
-1.75780

$\lcm{-1.75427}$

$\stackrel{\infty}{\perp}$ 


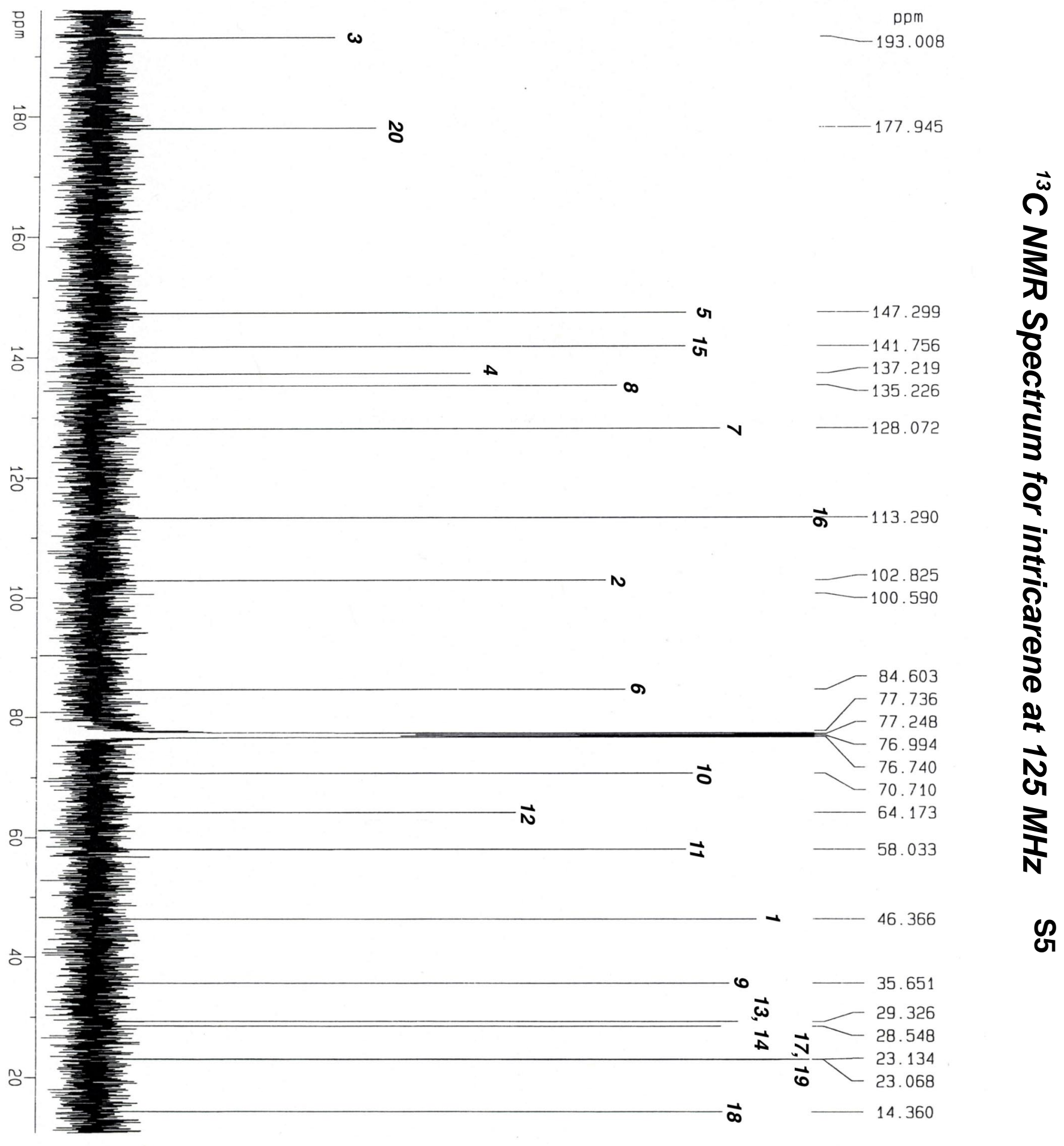




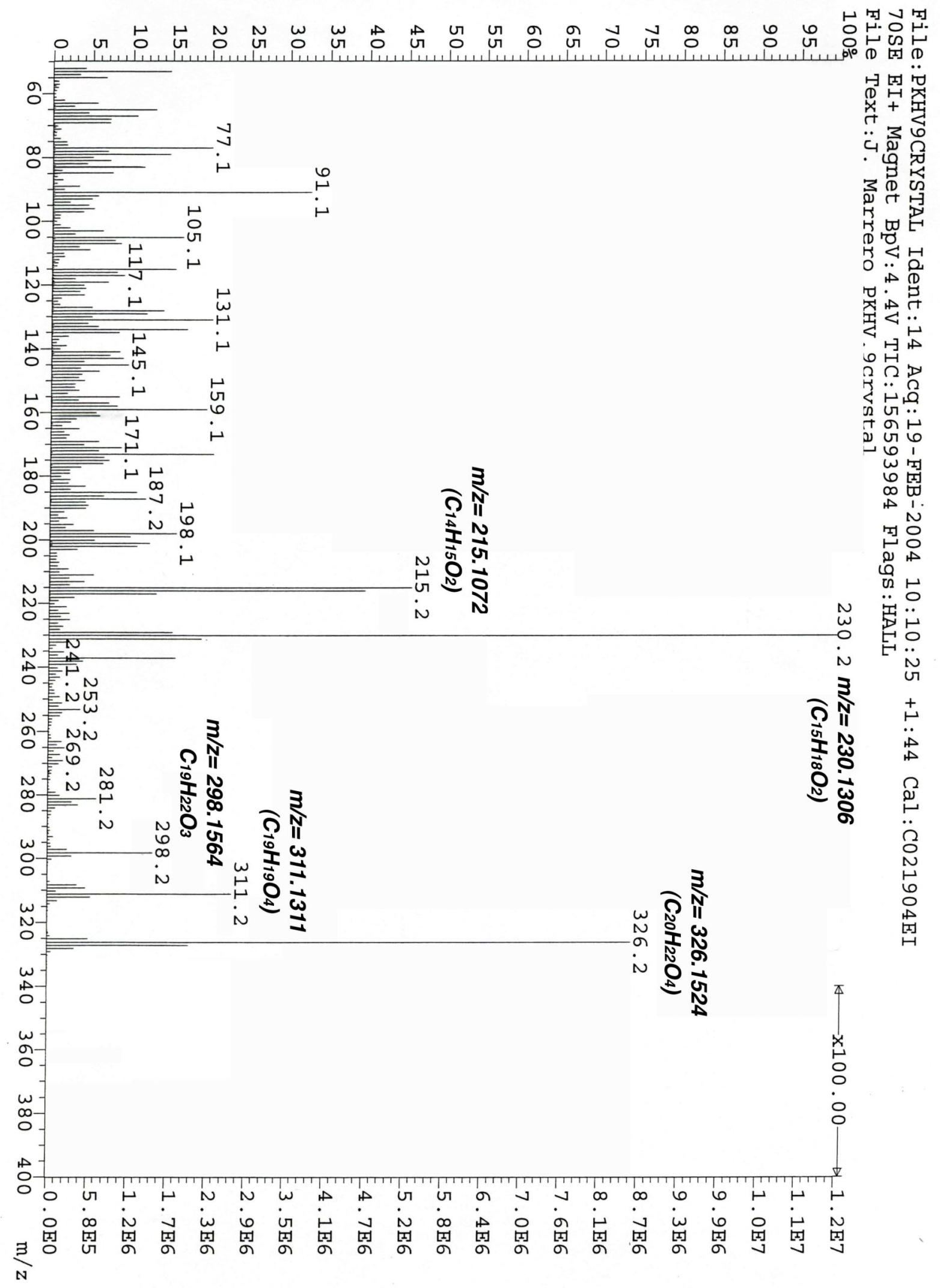

ค 


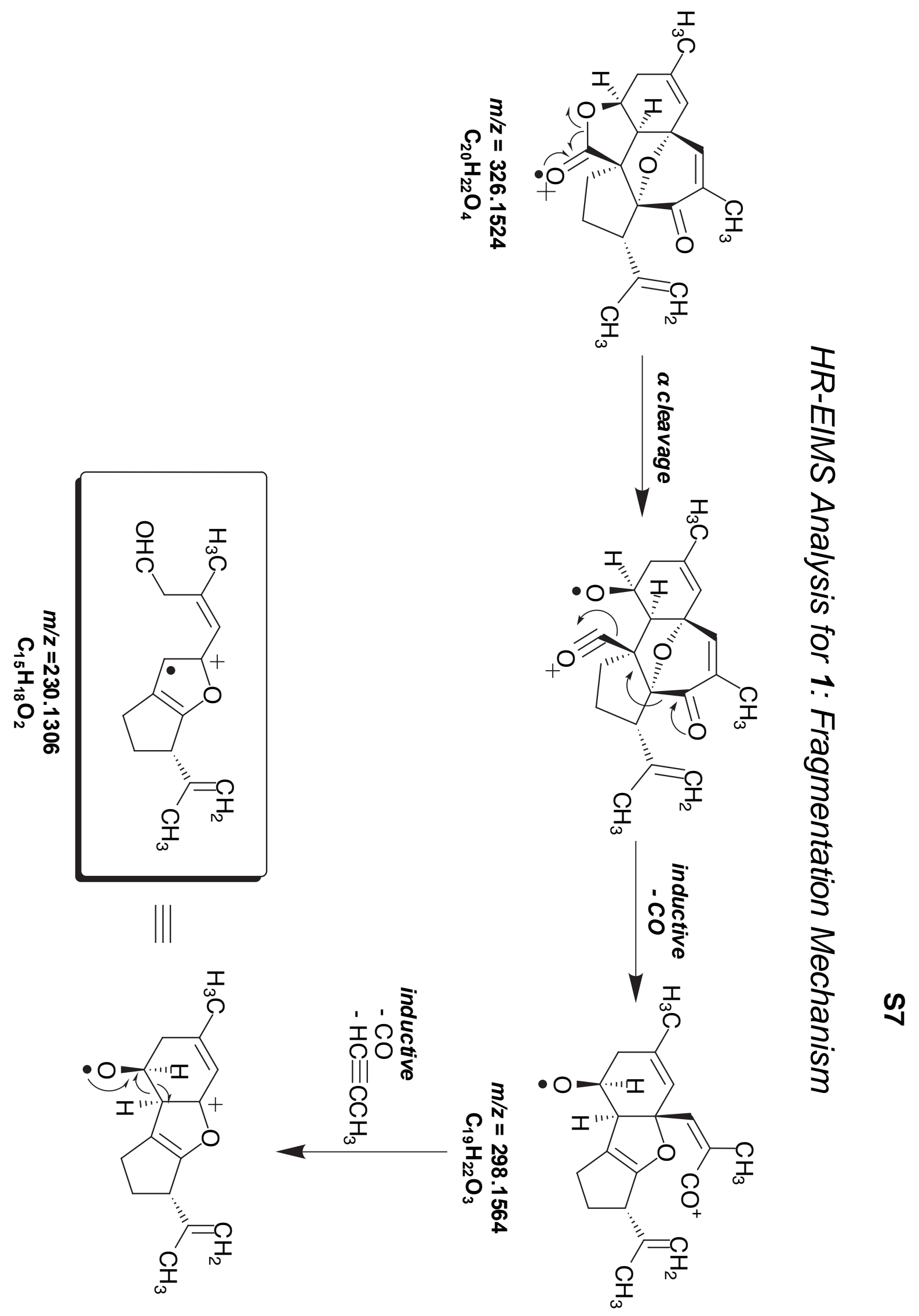


Table 1: Crystal data and structure refinement for intricarene (1).

\section{Intricarene}

Empirical formula

Formula weight

Temperature $(\mathrm{K})$

Wavelength $(\AA)$

Crystal system

Space group

Unit cell dimensions

$\mathrm{a}(\AA)$

b $(\AA)$

c (§)

$\alpha$ (deg)

$\beta$ (deg)

$\gamma(\operatorname{deg})$

Volume $\left(\AA^{3}\right)$

$\mathrm{Z}$

Density (calc.) $\left(\mathrm{Mg} \mathrm{m}^{-3}\right)$

Absorption coefficient $\left(\mathrm{mm}^{-1}\right)$

$\mathrm{F}(000)$

Crystal size (mm)

$\theta$ range for data collection (deg)

Limiting index

Reflections collected/unique

Completeness of $\theta=27.16$

Absorption correction

Max. and min. transmission

Refinement method

Data/restraints/parameters

Goodness-of-fit on $\mathrm{F}^{2}$

Final $R$ indices $[I>2 \sigma(I)]$

$R$ indices (all data)

Largest diff. peak and hole (e $\left.\AA^{-3}\right)$
$\mathrm{C}_{20} \mathrm{H}_{22} \mathrm{O}_{4}$

326.38

$173(2)$

0.71073

Monoclinic

$\mathrm{P} 2{ }_{1}$

7.1134(4)

12.6053(6)

9.4510(5)

90

$105.5470(2)$

90

816.43(7)

2

1.328

0.092

348

$0.50 \times 0.25 \times 0.25$

2.24 to 27.16

$-8 \leq h \leq 9,-16 \leq k \leq 14,-12 \leq l \leq 12$

$5909 / 1882[R($ int $)=0.0253]$

$99.5 \%$

Semi-empirical from equivalents

$0.98 / 0.96$

Full-matrix least-squares $F^{2}$

1882 / 1 / 220

1.030

$R 1=0.0322, w R 2=0.0854$

$R 1=0.0328, w R 2=0.0861$

0.277 and -0.132 
$\& \mathrm{~F}$

$\& W 64$

\&!Table 1. Atomic coordinates [ $\left.\mathrm{x} 10 \AA^{\wedge} 4 \& 0\right]$ and equivalent isotropic displacement parameters $\left[\& G A \&^{\wedge} 2 \& 0 \times 10 \& \wedge 3 \& 0\right]$ for ar035. U(eq) is defined as

one third of the trace of the orthogonalized \&IU\&vij\&O\&:\&! tensor.\&N $\& \mathrm{C}$

\begin{tabular}{|c|c|c|c|c|}
\hline \multicolumn{5}{|l|}{$\& E$} \\
\hline \multicolumn{5}{|l|}{ \& J2 } \\
\hline \multicolumn{5}{|l|}{$\& C$} \\
\hline & $\mathrm{x}$ & $\mathrm{Y}$ & $\mathrm{z}$ & $\mathrm{U}(\mathrm{eq})$ \\
\hline \multicolumn{5}{|l|}{$\& E$} \\
\hline \multicolumn{5}{|l|}{$£ C$} \\
\hline$O(1)$ & $8677(1)$ & $10597(1)$ & $11021(1)$ & $45(1)$ \\
\hline$O(2)$ & $7997(1)$ & $9586(1)$ & $9767(1)$ & $33(1)$ \\
\hline$O(3)$ & $7751(1)$ & $9762(1)$ & $5948(1)$ & $49(1)$ \\
\hline$O(4)$ & $4753(1)$ & $8636(1)$ & $7591(1)$ & $55(1)$ \\
\hline$O(5)$ & $4377(1)$ & 8581 (1) & $9324(1)$ & $56(1)$ \\
\hline$O(6)$ & $6773(1)$ & $7173(1)$ & $9188(1)$ & $47(1)$ \\
\hline$O(7)$ & $8507(2)$ & $6724(1)$ & $9995(2)$ & $65(1)$ \\
\hline$O(8)$ & $7318(2)$ & $8947(1)$ & $13971(1)$ & $70(1)$ \\
\hline$O(9)$ & $7220(2)$ & $9914(1)$ & $12541(1)$ & $57(1)$ \\
\hline C (1) & $6325(2)$ & $8880(1)$ & $11334(1)$ & $39(1)$ \\
\hline$C(2)$ & $7435(2)$ & $9437(1)$ & $11577(1)$ & $39(1)$ \\
\hline$C(3)$ & $7705(2)$ & $10082(1)$ & $10699(1)$ & $36(1)$ \\
\hline$C(4)$ & $6649(2)$ & $10613(1)$ & $10253(2)$ & $40(1)$ \\
\hline$C(5)$ & $6263(2)$ & $10316(1)$ & $9291(1)$ & $36(1)$ \\
\hline$C(6)$ & $7016(1)$ & $9585(1)$ & $8960(1)$ & $32(1)$ \\
\hline$C(7)$ & $7509(1)$ & $9486(1)$ & $7817(1)$ & $34(1)$ \\
\hline$C(8)$ & $7055(2)$ & $10028(1)$ & $6831(1)$ & $42(1)$ \\
\hline$C(9)$ & $5795(2)$ & $9686(2)$ & $6535(2)$ & $49(1)$ \\
\hline C (10) & $5759(2)$ & $8759(2)$ & $6923(2)$ & $46(1)$ \\
\hline C (11) & $6889(1)$ & $8608(1)$ & $7681(1)$ & $37(1)$ \\
\hline C (12) & $6451(1)$ & $8645(1)$ & $8832(1)$ & $34(1)$ \\
\hline C (13) & $6995(2)$ & $8017(1)$ & $9668(2)$ & $37(1)$ \\
\hline$C(14)$ & $6507(2)$ & $8009(1)$ & $10793(2)$ & $44(1)$ \\
\hline C (15) & $5866(2)$ & 8791 (2) & $12454(2)$ & $49(1)$ \\
\hline C (16) & $6569(2)$ & $9395(2)$ & $13216(2)$ & $56(1)$ \\
\hline C (17) & $4989(2)$ & $8302(2)$ & $12730(2)$ & $75(1)$ \\
\hline$C(18)$ & $6189(2)$ & $11349(2)$ & $10876(2)$ & $61(1)$ \\
\hline C (19) & $7141(2)$ & $10991(2)$ & $6956(2)$ & $54(1)$ \\
\hline$C(20)$ & $5101(2)$ & $8617(1)$ & $8655(2)$ & $41(1)$ \\
\hline C (21) & $7615(2)$ & $6582(1)$ & $9410(2)$ & $50(1)$ \\
\hline$C(22)$ & $7316(4)$ & $5752(2)$ & $8866(3)$ & $85(1)$ \\
\hline$O(51)$ & $251(1)$ & $10874(1)$ & $6883(1)$ & $51(1)$ \\
\hline$O(52)$ & $401(1)$ & $9630(1)$ & $5919(1)$ & $36(1)$ \\
\hline$O(53)$ & $-820(2)$ & $6849(1)$ & $6788(1)$ & $65(1)$ \\
\hline$O(54)$ & $2685(1)$ & $7250(1)$ & $5410(1)$ & $55(1)$ \\
\hline$O(55)$ & $3687(1)$ & $8431(1)$ & $5063(1)$ & $51(1)$ \\
\hline$O(56)$ & $1405(1)$ & $8490(1)$ & $3118(1)$ & $47(1)$ \\
\hline$O(57)$ & $347(2)$ & $9409(1)$ & $2053(2)$ & $70(1)$ \\
\hline$O(58)$ & $2788(2)$ & $12485(1)$ & $4737(1)$ & $60(1)$ \\
\hline$O(59)$ & $2273(2)$ & $11526(1)$ & $6053(1)$ & $59(1)$ \\
\hline$C(51)$ & $2567(2)$ & $10324(1)$ & $4955(1)$ & $37(1)$ \\
\hline C ( 52$)$ & $1631(2)$ & $10823(1)$ & $5545(2)$ & $41(1)$ \\
\hline$C(53)$ & $1064(2)$ & $10330(1)$ & $6445(1)$ & $37(1)$ \\
\hline
\end{tabular}




\begin{tabular}{lrrrr}
$C(54)$ & $1950(2)$ & $9886(1)$ & $7236(1)$ & $38(1)$ \\
$C(55)$ & $1926(2)$ & $9055(1)$ & $7038(1)$ & $36(1)$ \\
$C(56)$ & $1033(1)$ & $8846(1)$ & $6118(1)$ & $32(1)$ \\
$C(57)$ & $123(2)$ & $8092(1)$ & $6119(1)$ & $37(1)$ \\
$C(58)$ & $194(2)$ & $7402(1)$ & $6999(2)$ & $44(1)$ \\
$C(59)$ & $1308(3)$ & $6882(2)$ & $6772(2)$ & $61(1)$ \\
$C(60)$ & $1470(2)$ & $6973(1)$ & $5556(2)$ & $49(1)$ \\
$C(61)$ & $649(2)$ & $7706(1)$ & $5114(2)$ & $41(1)$ \\
$C(62)$ & $1505(2)$ & $8468(1)$ & $5027(1)$ & $35(1)$ \\
$C(63)$ & $1290(2)$ & $9060(1)$ & $4049(1)$ & $37(1)$ \\
$C(64)$ & $2138(2)$ & $9815(1)$ & $3942(2)$ & $42(1)$ \\
$C(65)$ & $3419(2)$ & $11034(1)$ & $4692(2)$ & $45(1)$ \\
$C(66)$ & $3171(2)$ & $11791(1)$ & $5397(2)$ & $49(1)$ \\
$C(67)$ & $4225(2)$ & $11035(2)$ & $3977(2)$ & $65(1)$ \\
$C(68)$ & $2742(2)$ & $10367(2)$ & $8056(2)$ & $51(1)$ \\
$C(69)$ & $214(2)$ & $7711(2)$ & $8166(2)$ & $50(1)$ \\
$C(70)$ & $2748(2)$ & $8086(1)$ & $5155(1)$ & $40(1)$ \\
$C(71)$ & $872(2)$ & $8744(2)$ & $2169(2)$ & $58(1)$ \\
$C(72)$ & $1050(5)$ & $8106(3)$ & $1289(2)$ & $115(2)$ \\
O (91) & $9980(2)$ & $542(2)$ & $8964(2)$ & $110(1)$ \\
O (92) & $8895(9)$ & $7728(7)$ & $2642(11)$ & $90(4)$ \\
\hline \& & & & & \\
\&J2 & & & & \\
\& F & & & &
\end{tabular}

\begin{tabular}{ll}
\hline$\& E$ & \\
$\& D$ & \\
$O(1)-C(3)$ & $1.391(2)$ \\
$O(2)-C(6)$ & $1.4300(19)$ \\
$O(2)-C(3)$ & $1.448(2)$ \\
$O(3)-C(8)$ & $1.454(2)$ \\
$O(4)-C(20)$ & $1.345(2)$ \\
$O(4)-C(10)$ & $1.464(2)$ \\
$O(5)-C(20)$ & $1.205(2)$ \\
$O(6)-C(21)$ & $1.337(3)$ \\
$O(6)-C(13)$ & $1.461(2)$ \\
$O(7)-C(21)$ & $1.210(3)$ \\
$O(8)-C(16)$ & $1.397(3)$ \\
$O(9)-C(16)$ & $1.410(3)$ \\
$O(9)-C(2)$ & $1.443(2)$ \\
$C(1)-C(15)$ & $1.522(2)$ \\
$C(1)-C(2)$ & $1.533(3)$ \\
$C(1)-C(14)$ & $1.539(3)$ \\
$C(2)-C(3)$ & $1.529(2)$ \\
$C(3)-C(4)$ & $1.518(3)$ \\
$C(4)-C(5)$ & $1.321(3)$ \\
$C(4)-C(18)$ & $1.500(3)$ \\
$C(5)-C(6)$ & $1.498(2)$ \\
$C(6)-C(7)$ & $1.566(2)$ \\
$C(6)-C(12)$ & $1.604(2)$ \\
$C(7)-C(8)$ & $1.540(2)$ \\
$C(7)-C(11)$ & $1.543(3)$ \\
$C(8)-C(19)$ & $1.516(3)$ \\
$C(8)-C(9)$ & $1.533(3)$ \\
$C(9)-C(10)$ & $1.528(3)$ \\
& \\
\hline &
\end{tabular}




\begin{tabular}{|c|c|}
\hline$C(10)-C(11)$ & $1.538(2)$ \\
\hline$C(11)-C(12)$ & $1.544(2)$ \\
\hline$C(12)-C(20)$ & $1.518(2)$ \\
\hline$C(12)-C(13)$ & $1.521(2)$ \\
\hline$C(13)-C(14)$ & $1.535(3)$ \\
\hline$C(15)-C(17)$ & $1.313(4)$ \\
\hline$C(15)-C(16)$ & $1.514(3)$ \\
\hline$C(21)-C(22)$ & $1.489(4)$ \\
\hline$O(51)-C(53)$ & $1.390(2)$ \\
\hline$O(52)-C(56)$ & $1.429(2)$ \\
\hline$O(52)-C(53)$ & $1.450(2)$ \\
\hline$O(53)-C(58)$ & $1.439(3)$ \\
\hline $\mathrm{O}(54)-\mathrm{C}(70)$ & $1.347(3)$ \\
\hline$O(54)-C(60)$ & $1.457(3)$ \\
\hline$O(55)-C(70)$ & $1.199(3)$ \\
\hline$O(56)-C(71)$ & $1.336(3)$ \\
\hline$O(56)-C(63)$ & $1.470(2)$ \\
\hline $\mathrm{O}(57)-\mathrm{C}(71)$ & $1.199(3)$ \\
\hline$O(58)-C(66)$ & $1.406(3)$ \\
\hline $\mathrm{O}(59)-\mathrm{C}(66)$ & $1.406(2)$ \\
\hline$O(59)-C(52)$ & $1.433(2)$ \\
\hline$C(51)-C(65)$ & $1.517(3)$ \\
\hline$C(51)-C(64)$ & $1.532(3)$ \\
\hline$C(51)-C(52)$ & $1.538(2)$ \\
\hline$C(52)-C(53)$ & $1.533(2)$ \\
\hline$C(53)-C(54)$ & $1.510(3)$ \\
\hline$C(54)-C(55)$ & $1.322(3)$ \\
\hline$C(54)-C(68)$ & $1.500(3)$ \\
\hline$C(55)-C(56)$ & $1.494(2)$ \\
\hline$C(56)-C(57)$ & $1.562(3)$ \\
\hline$C(56)-C(62)$ & $1.602(2)$ \\
\hline$C(57)-C(58)$ & $1.531(3)$ \\
\hline$C(57)-C(61)$ & $1.542(2)$ \\
\hline$C(58)-C(69)$ & $1.521(3)$ \\
\hline$C(58)-C(59)$ & $1.538(3)$ \\
\hline$C(59)-C(60)$ & $1.536(3)$ \\
\hline$C(60)-C(61)$ & $1.545(3)$ \\
\hline$C(61)-C(62)$ & $1.541(3)$ \\
\hline$C(62)-C(70)$ & $1.517(2)$ \\
\hline$C(62)-C(63)$ & $1.528(2)$ \\
\hline$C(63)-C(64)$ & $1.529(3)$ \\
\hline$C(65)-C(67)$ & $1.315(3)$ \\
\hline$C(65)-C(66)$ & $1.510(3)$ \\
\hline$C(71)-C(72)$ & $1.502(4)$ \\
\hline
\end{tabular}

$\& E$

$\& D$

$\mathrm{C}(6)-\mathrm{O}(2)-\mathrm{C}(3)$

$110.28(12)$

$\mathrm{C}(20)-\mathrm{O}(4)-\mathrm{C}(10)$

$\mathrm{C}(21)-\mathrm{O}(6)-\mathrm{C}(13)$

$C(16)-O(9)-C(2)$

$C(15)-C(1)-C(2)$

$C(15)-C(1)-C(14)$

$\mathrm{C}(2)-\mathrm{C}(1)-\mathrm{C}(14)$

$\mathrm{O}(9)-\mathrm{C}(2)-\mathrm{C}(3)$

$\mathrm{O}(9)-\mathrm{C}(2)-\mathrm{C}(1)$

$\mathrm{C}(3)-\mathrm{C}(2)-\mathrm{C}(1)$

$\mathrm{O}(1)-\mathrm{C}(3)-\mathrm{O}(2)$

$O(1)-C(3)-C(4)$

$\mathrm{O}(2)-\mathrm{C}(3)-\mathrm{C}(4)$

$O(1)-C(3)-C(2)$
$112.16(14)$

$116.44(16)$

$108.71(16)$

101.65 ( 16$)$

$112.38(16)$

$117.04(15)$

$107.59(15)$

$105.64(15)$

115.98 (15)

$108.47(14)$

111.21 (15)

102.91 (13)

111.85 (14) 


$$
\begin{aligned}
& \mathrm{O}(2)-\mathrm{C}(3)-\mathrm{C}(2) \\
& C(4)-C(3)-C(2) \\
& C(5)-C(4)-C(18) \\
& C(5)-C(4)-C(3) \\
& C(18)-C(4)-C(3) \\
& C(4)-C(5)-C(6) \\
& O(2)-C(6)-C(5) \\
& O(2)-C(6)-C(7) \\
& C(5)-C(6)-C(7) \\
& \mathrm{O}(2)-\mathrm{C}(6)-\mathrm{C}(12) \\
& C(5)-C(6)-C(12) \\
& C(7)-C(6)-C(12) \\
& C(8)-C(7)-C(11) \\
& C(8)-C(7)-C(6) \\
& C(11)-C(7)-C(6) \\
& \mathrm{O}(3)-\mathrm{C}(8)-\mathrm{C}(19) \\
& \mathrm{O}(3)-\mathrm{C}(8)-\mathrm{C}(9) \\
& C(19)-C(8)-C(9) \\
& \mathrm{O}(3)-\mathrm{C}(8)-\mathrm{C}(7) \\
& C(19)-C(8)-C(7) \\
& \mathrm{C}(9)-\mathrm{C}(8)-\mathrm{C}(7) \\
& C(10)-C(9)-C(8) \\
& O(4)-C(10)-C(9) \\
& \mathrm{O}(4)-\mathrm{C}(10)-\mathrm{C}(11) \\
& \mathrm{C}(9)-\mathrm{C}(10)-\mathrm{C}(11) \\
& C(10)-C(11)-C(7) \\
& C(10)-C(11)-C(12) \\
& C(7)-C(11)-C(12) \\
& C(20)-C(12)-C(13) \\
& C(20)-C(12)-C(11) \\
& C(13)-C(12)-C(11) \\
& C(20)-C(12)-C(6) \\
& C(13)-C(12)-C(6) \\
& C(11)-C(12)-C(6) \\
& \mathrm{O}(6)-\mathrm{C}(13)-\mathrm{C}(12) \\
& \mathrm{O}(6)-\mathrm{C}(13)-\mathrm{C}(14) \\
& \mathrm{C}(12)-\mathrm{C}(13)-\mathrm{C}(14) \\
& C(13)-C(14)-C(1) \\
& C(17)-C(15)-C(16) \\
& C(17)-C(15)-C(1) \\
& C(16)-C(15)-C(1) \\
& \mathrm{O}(8)-\mathrm{C}(16)-\mathrm{O}(9) \\
& \mathrm{O}(8)-\mathrm{C}(16)-\mathrm{C}(15) \\
& \mathrm{O}(9)-\mathrm{C}(16)-\mathrm{C}(15) \\
& \mathrm{O}(5)-\mathrm{C}(20)-\mathrm{O}(4) \\
& \mathrm{O}(5)-\mathrm{C}(20)-\mathrm{C}(12) \\
& \mathrm{O}(4)-\mathrm{C}(20)-\mathrm{C}(12) \\
& \mathrm{O}(7)-\mathrm{C}(21)-\mathrm{O}(6) \\
& \mathrm{O}(7)-\mathrm{C}(21)-\mathrm{C}(22) \\
& \mathrm{O}(6)-\mathrm{C}(21)-\mathrm{C}(22) \\
& C(56)-O(52)-C(53) \\
& C(70)-O(54)-C(60) \\
& C(71)-O(56)-C(63) \\
& C(66)-O(59)-C(52) \\
& C(65)-C(51)-C(64) \\
& C(65)-C(51)-C(52) \\
& C(64)-C(51)-C(52) \\
& O(59)-C(52)-C(53) \\
& O(59)-C(52)-C(51) \\
& \text { C ( } 53)-C(52)-C(51)
\end{aligned}
$$




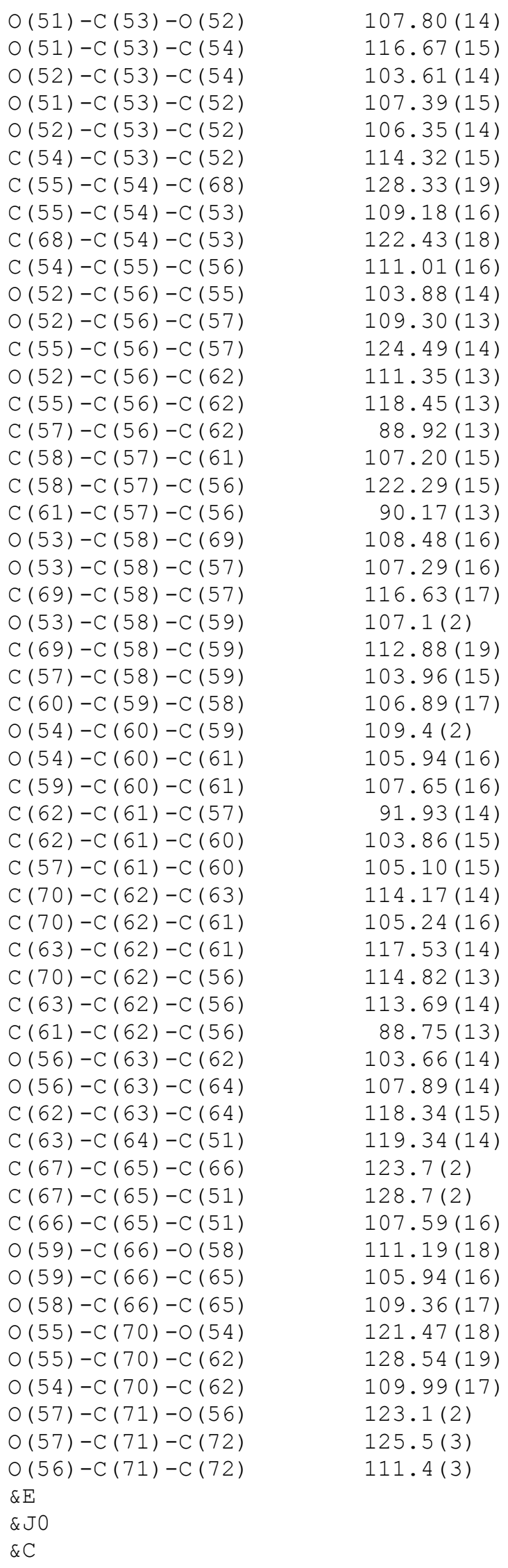

$\& \mathrm{C}$ 


\begin{tabular}{|c|c|c|c|c|c|c|}
\hline \multicolumn{7}{|l|}{$\& E$} \\
\hline \multicolumn{7}{|l|}{ \& J3 } \\
\hline \multicolumn{7}{|l|}{$\&$ HSy } \\
\hline \multicolumn{7}{|l|}{$\& \mathrm{E}$} \\
\hline \multicolumn{7}{|l|}{$\& \mathrm{~F}$} \\
\hline \multicolumn{7}{|c|}{ \&W72 } \\
\hline \multicolumn{7}{|c|}{ 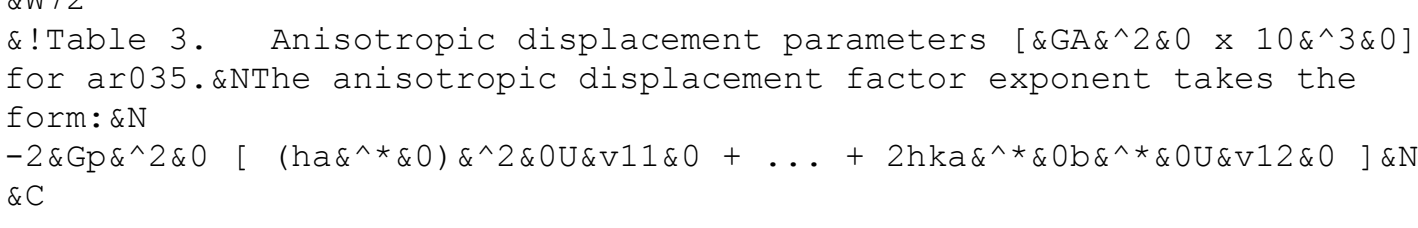 } \\
\hline \multicolumn{7}{|l|}{$\& E$} \\
\hline \multicolumn{7}{|l|}{$\& \mathrm{~J} 2$} \\
\hline \multicolumn{7}{|l|}{$\& \mathrm{C}$} \\
\hline & U11 & $\mathrm{U} 22$ & U33 & $\mathrm{U} 23$ & U13 & $\mathrm{U} 12$ \\
\hline \multicolumn{7}{|l|}{$\& E$} \\
\hline \multicolumn{7}{|l|}{$\& \mathrm{C}$} \\
\hline$O(1)$ & $50(1)$ & $48(1)$ & 37 (1) & $-2(1)$ & $-5(1)$ & $-5(1)$ \\
\hline$O(2)$ & $31(1)$ & $39(1)$ & $28(1)$ & $-1(1)$ & $-1(1)$ & $3(1)$ \\
\hline$O(3)$ & $40(1)$ & $80(1)$ & $29(1)$ & $-2(1)$ & $3(1)$ & $2(1)$ \\
\hline$O(4)$ & $30(1)$ & $83(1)$ & $50(1)$ & $2(1)$ & $-3(1)$ & $-8(1)$ \\
\hline$O(5)$ & $33(1)$ & $74(1)$ & $61(1)$ & $6(1)$ & $10(1)$ & $-1(1)$ \\
\hline$O(6)$ & $51(1)$ & $39(1)$ & $50(1)$ & $-5(1)$ & $0(1)$ & $5(1)$ \\
\hline$O(7)$ & $63(1)$ & $64(1)$ & $70(1)$ & $18(1)$ & 7 (1) & $22(1)$ \\
\hline$O(8)$ & $104(1)$ & $60(1)$ & $43(1)$ & $7(1)$ & $-3(1)$ & $4(1)$ \\
\hline$O(9)$ & $90(1)$ & $53(1)$ & $30(1)$ & $-3(1)$ & $11(1)$ & $-7(1)$ \\
\hline$C(1)$ & 41 (1) & $44(1)$ & $34(1)$ & $5(1)$ & $8(1)$ & $5(1)$ \\
\hline$C(2)$ & $47(1)$ & $41(1)$ & $29(1)$ & $2(1)$ & $2(1)$ & $4(1)$ \\
\hline C (3) & $39(1)$ & $38(1)$ & $30(1)$ & $-2(1)$ & $3(1)$ & $1(1)$ \\
\hline C (4) & $44(1)$ & $39(1)$ & $36(1)$ & $3(1)$ & $6(1)$ & $6(1)$ \\
\hline C ( 5$)$ & $35(1)$ & $38(1)$ & $36(1)$ & $4(1)$ & $3(1)$ & $5(1)$ \\
\hline$C(6)$ & $29(1)$ & $38(1)$ & $29(1)$ & $2(1)$ & $0(1)$ & $2(1)$ \\
\hline$C(7)$ & $29(1)$ & $46(1)$ & $29(1)$ & $-2(1)$ & $2(1)$ & $3(1)$ \\
\hline C ( 8$)$ & $41(1)$ & $56(1)$ & $29(1)$ & $2(1)$ & $4(1)$ & $5(1)$ \\
\hline C (9) & $35(1)$ & $73(1)$ & $38(1)$ & $7(1)$ & $-2(1)$ & $10(1)$ \\
\hline$C(10)$ & $34(1)$ & $67(1)$ & $35(1)$ & $-8(1)$ & $-1(1)$ & $1(1)$ \\
\hline C (11) & 31 (1) & $45(1)$ & $35(1)$ & $-7(1)$ & $2(1)$ & $3(1)$ \\
\hline C (12) & $29(1)$ & $38(1)$ & $35(1)$ & $-3(1)$ & $2(1)$ & $2(1)$ \\
\hline C (13) & 35 (1) & $35(1)$ & $40(1)$ & $-1(1)$ & $3(1)$ & $1(1)$ \\
\hline C (14) & $51(1)$ & $39(1)$ & $43(1)$ & $4(1)$ & $8(1)$ & $-3(1)$ \\
\hline$C(15)$ & $51(1)$ & $59(1)$ & $41(1)$ & $7(1)$ & $14(1)$ & $9(1)$ \\
\hline C (16) & $77(1)$ & $56(1)$ & $38(1)$ & $2(1)$ & $14(1)$ & $10(1)$ \\
\hline C (17) & $67(2)$ & $106(2)$ & $56(1)$ & $3(1)$ & $29(1)$ & $-12(2)$ \\
\hline $\mathrm{C}(18)$ & $77(2)$ & $57(1)$ & $50(1)$ & $-10(1)$ & $4(1)$ & $23(1)$ \\
\hline C (19) & $63(1)$ & $55(1)$ & $46(1)$ & $12(1)$ & $10(1)$ & $8(1)$ \\
\hline$C(20)$ & $32(1)$ & $43(1)$ & $48(1)$ & $0(1)$ & $2(1)$ & $-1(1)$ \\
\hline$C(21)$ & $68(1)$ & $44(1)$ & $40(1)$ & $8(1)$ & $19(1)$ & $16(1)$ \\
\hline C (22) & $139(3)$ & $52(2)$ & $64(2)$ & $-15(1)$ & $4(2)$ & $29(2)$ \\
\hline O (51) & $55(1)$ & $48(1)$ & $51(1)$ & $2(1)$ & $21(1)$ & $14(1)$ \\
\hline $\mathrm{O}(52)$ & $34(1)$ & $37(1)$ & $38(1)$ & $4(1)$ & $3(1)$ & $4(1)$ \\
\hline$O(53)$ & 85 (1) & $49(1)$ & $60(1)$ & $12(1)$ & $1(1)$ & $-21(1)$ \\
\hline O (54) & $59(1)$ & $51(1)$ & $56(1)$ & $0(1)$ & $12(1)$ & $19(1)$ \\
\hline $\mathrm{O}(55)$ & $39(1)$ & $68(1)$ & $45(1)$ & $-5(1)$ & $6(1)$ & $9(1)$ \\
\hline$O(56)$ & $58(1)$ & $52(1)$ & $29(1)$ & $-2(1)$ & $-2(1)$ & $1(1)$ \\
\hline$O(57)$ & $63(1)$ & $87(1)$ & $57(1)$ & $22(1)$ & $-19(1)$ & $-4(1)$ \\
\hline O (58) & $77(1)$ & $44(1)$ & $61(1)$ & $1(1)$ & $13(1)$ & $-4(1)$ \\
\hline
\end{tabular}




\begin{tabular}{|c|c|c|c|c|c|c|}
\hline O (59) & $77(1)$ & $46(1)$ & $56(1)$ & $-9(1)$ & $27(1)$ & $-15(1)$ \\
\hline C (51) & $37(1)$ & $40(1)$ & $35(1)$ & $2(1)$ & $7(1)$ & $0(1)$ \\
\hline C (52) & $45(1)$ & $38(1)$ & $40(1)$ & $3(1)$ & $9(1)$ & $3(1)$ \\
\hline C (53) & $38(1)$ & $40(1)$ & $35(1)$ & $1(1)$ & $8(1)$ & $3(1)$ \\
\hline C (54) & $37(1)$ & $48(1)$ & $30(1)$ & $-2(1)$ & $7(1)$ & $2(1)$ \\
\hline C (55) & $32(1)$ & $46(1)$ & $29(1)$ & $3(1)$ & $6(1)$ & $6(1)$ \\
\hline$C(56)$ & $32(1)$ & $37(1)$ & $29(1)$ & $4(1)$ & $5(1)$ & $6(1)$ \\
\hline C (57) & $37(1)$ & $38(1)$ & $36(1)$ & $4(1)$ & $4(1)$ & $2(1)$ \\
\hline$C(58)$ & $55(1)$ & $39(1)$ & $37(1)$ & $6(1)$ & $9(1)$ & $2(1)$ \\
\hline C (59) & $84(2)$ & $53(1)$ & $47(1)$ & $15(1)$ & $16(1)$ & $27(1)$ \\
\hline$C(60)$ & $64(1)$ & $40(1)$ & $44(1)$ & $1(1)$ & $12(1)$ & $8(1)$ \\
\hline C (61) & $48(1)$ & $42(1)$ & $33(1)$ & $2(1)$ & $1(1)$ & $-3(1)$ \\
\hline$C(62)$ & $36(1)$ & $42(1)$ & $27(1)$ & $0(1)$ & $2(1)$ & $2(1)$ \\
\hline C (63) & $40(1)$ & $45(1)$ & $26(1)$ & $3(1)$ & $2(1)$ & $2(1)$ \\
\hline$C(64)$ & $50(1)$ & $46(1)$ & $31(1)$ & $3(1)$ & $10(1)$ & $-3(1)$ \\
\hline C (65) & $39(1)$ & $47(1)$ & $48(1)$ & $7(1)$ & $5(1)$ & $0(1)$ \\
\hline C (66) & $50(1)$ & $49(1)$ & $49(1)$ & $-3(1)$ & $8(1)$ & $-10(1)$ \\
\hline C (67) & $56(1)$ & $58(1)$ & $84(2)$ & $4(1)$ & $29(1)$ & $-6(1)$ \\
\hline$C(68)$ & $50(1)$ & $59(1)$ & $44(1)$ & $-13(1)$ & $-1(1)$ & $0(1)$ \\
\hline C (69) & $63(1)$ & $52(1)$ & $37(1)$ & $6(1)$ & $13(1)$ & $0(1)$ \\
\hline$C(70)$ & $45(1)$ & $50(1)$ & $25(1)$ & $-3(1)$ & $4(1)$ & $12(1)$ \\
\hline C (71) & $63(1)$ & $77(2)$ & $33(1)$ & $5(1)$ & $-7(1)$ & $-13(1)$ \\
\hline C (72) & $174(4)$ & $124(3)$ & $42(1)$ & $-25(2)$ & $-14(2)$ & $-5(3)$ \\
\hline$O(91)$ & $104(2)$ & $178(3)$ & $52(1)$ & $-38(1)$ & $33(1)$ & $-92(2)$ \\
\hline O (92) & $67(6)$ & $79(7)$ & $122(9)$ & $60(7)$ & $-3(6)$ & $-2(5)$ \\
\hline
\end{tabular}

\begin{tabular}{l}
\hline $\mathrm{E}$ \\
$\& \mathrm{~J} 3$ \\
$\& \mathrm{~F}$
\end{tabular}

\&W64

\&!Table 4. Hydrogen coordinates $\left(\times 10 x^{\wedge} 4 \& 0\right)$ and isotropic displacement parameters $(\& G A \& \wedge 2 \& 0 \times 10 \& \wedge 3 \& 0)$ for $\operatorname{ar} 035 . \& \mathrm{H}$ $\& \mathrm{C}$

\begin{tabular}{lllll}
\hline$\& \mathrm{E}$ & & & & \\
$\& \mathrm{~J} 2$ & & & & \\
$\& \mathrm{C}$ & $\mathrm{x}$ & $\mathrm{y}$ & $\mathrm{U}(\mathrm{eq})$ \\
\end{tabular}

$\& E$

$\& \mathrm{C}$

$\begin{array}{lrrrr}\text { H (1) } & 9201 & 10305 & 11337 & 68 \\ \text { H (3) } & 8451 & 9900 & 6083 & 74 \\ \text { H (8) } & 7442 & 9236 & 14521 & 104 \\ \text { H (1A) } & 5740 & 9208 & 10875 & 47 \\ \text { H (2) } & 8131 & 9067 & 11730 & 47 \\ \text { H } 5) & 5608 & 10532 & 8872 & 44 \\ \text { H (7) } & 8380 & 9431 & 7870 & 41 \\ \text { H (9A) } & 5213 & 10026 & 6883 & 58 \\ \text { H (9B) } & 5611 & 9713 & 5757 & 58 \\ \text { H (10) } & 5718 & 8361 & 6310 & 55 \\ \text { H (11) } & 7391 & 8120 & 7514 & 44 \\ \text { H (13) } & 7858 & 8113 & 9757 & 44 \\ \text { H (14A) } & 7047 & 7674 & 11273 & 53 \\ \text { H (14B) } & 5746 & 7713 & 10732 & 53 \\ \text { H (16) } & 6018 & 9750 & 13597 & 67 \\ \text { H (17A) } & 4763 & 8312 & 13437 & 90 \\ \text { H (17B) } & 4595 & 7945 & 12218 & 90 \\ \text { H (18A) } & 5488 & 11579 & 10489 & 92\end{array}$


S16

\begin{tabular}{|c|c|c|c|c|}
\hline $\mathrm{H}(18 \mathrm{~B})$ & 6790 & 11785 & 10962 & 92 \\
\hline $\mathrm{H}(18 \mathrm{C})$ & 5993 & 11155 & 11577 & 92 \\
\hline $\mathrm{H}(19 \mathrm{~A})$ & 6824 & 11260 & 6299 & 81 \\
\hline $\mathrm{H}(19 \mathrm{~B})$ & 7962 & 11152 & 7102 & 81 \\
\hline $\mathrm{H}(19 \mathrm{C})$ & 6693 & 11169 & 7546 & 81 \\
\hline $\mathrm{H}(22 \mathrm{~A})$ & 7951 & 5351 & 9031 & 128 \\
\hline $\mathrm{H}(22 \mathrm{~B})$ & 7212 & 5837 & 8096 & 128 \\
\hline $\mathrm{H}(22 \mathrm{C})$ & 6591 & 5531 & 9120 & 128 \\
\hline H (51) & 175 & 10739 & 7515 & 76 \\
\hline H ( 53$)$ & -1361 & 7115 & 6456 & 97 \\
\hline H ( 58$)$ & 2858 & 12929 & 5089 & 90 \\
\hline $\mathrm{H}(51 \mathrm{~A})$ & 2986 & 9934 & 5473 & 44 \\
\hline $\mathrm{H}(52)$ & 1007 & 11037 & 5018 & 49 \\
\hline H ( 55) & 2401 & 8654 & 7421 & 43 \\
\hline H (57) & -698 & 8290 & 5953 & 44 \\
\hline $\mathrm{H}(59 \mathrm{~A})$ & 2003 & 7100 & 7197 & 73 \\
\hline H (59B) & 1201 & 6286 & 6958 & 73 \\
\hline $\mathrm{H}(60)$ & 1283 & 6437 & 5170 & 58 \\
\hline $\mathrm{H}(61)$ & 95 & 7581 & 4483 & 49 \\
\hline H (63) & 470 & 9275 & 4025 & 44 \\
\hline $\mathrm{H}(64 \mathrm{~A})$ & 1749 & 10213 & 3427 & 50 \\
\hline $\mathrm{H}(64 \mathrm{~B})$ & 2839 & 9600 & 3621 & 50 \\
\hline H $(66)$ & 3892 & 11946 & 5850 & 59 \\
\hline $\mathrm{H}(67 \mathrm{~A})$ & 4681 & 11522 & 3883 & 78 \\
\hline $\mathrm{H}(67 \mathrm{~B})$ & 4339 & 10549 & 3564 & 78 \\
\hline $\mathrm{H}(68 \mathrm{~A})$ & 3385 & 10625 & 7706 & 77 \\
\hline $\mathrm{H}(68 \mathrm{~B})$ & 3061 & 9979 & 8606 & 77 \\
\hline $\mathrm{H}(68 \mathrm{C})$ & 2288 & 10804 & 8381 & 77 \\
\hline $\mathrm{H}(69 \mathrm{~A})$ & 237 & 7227 & 8644 & 75 \\
\hline $\mathrm{H}(69 \mathrm{~B})$ & -489 & 8042 & 8260 & 75 \\
\hline $\mathrm{H}(69 \mathrm{C})$ & 909 & 8058 & 8330 & 75 \\
\hline $\mathrm{H}(72 \mathrm{~A})$ & 1868 & 8116 & 1116 & 172 \\
\hline $\mathrm{H}(72 \mathrm{~B})$ & 543 & 8249 & 655 & 172 \\
\hline $\mathrm{H}(72 \mathrm{C})$ & 853 & 7544 & 1532 & 172 \\
\hline $\mathrm{H}(101)$ & 9376 & 382 & 9255 & 165 \\
\hline $\mathrm{H}(201)$ & 10269 & 977 & 9296 & 165 \\
\hline
\end{tabular}

